

En el paradigma actual de libre mercado, las empresas son capaces de tener un impacto significativo en los sistemas ambientales, sociales y financieros, a menudo a escala global. Después de la Cumbre de la Tierra de Río de Janeiro, en 1992, se realizaron varios esfuerzos para ayudar a las organizaciones a ser más sostenibles, tanto a nivel teórico como práctico. Conceptos como la sostenibilidad corporativa (CS) o la responsabilidad social empresarial (RSE) han sido adoptados por las empresas de todo el mundo, y las prácticas, como la gestión ambiental (EM) y los informes de RSE y de sostenibilidad, se implementan ampliamente.

Con la aprobación de los ODS, la comunidad empresarial ha cobrado un protagonismo sin precedente, porque los retos de nuestro planeta
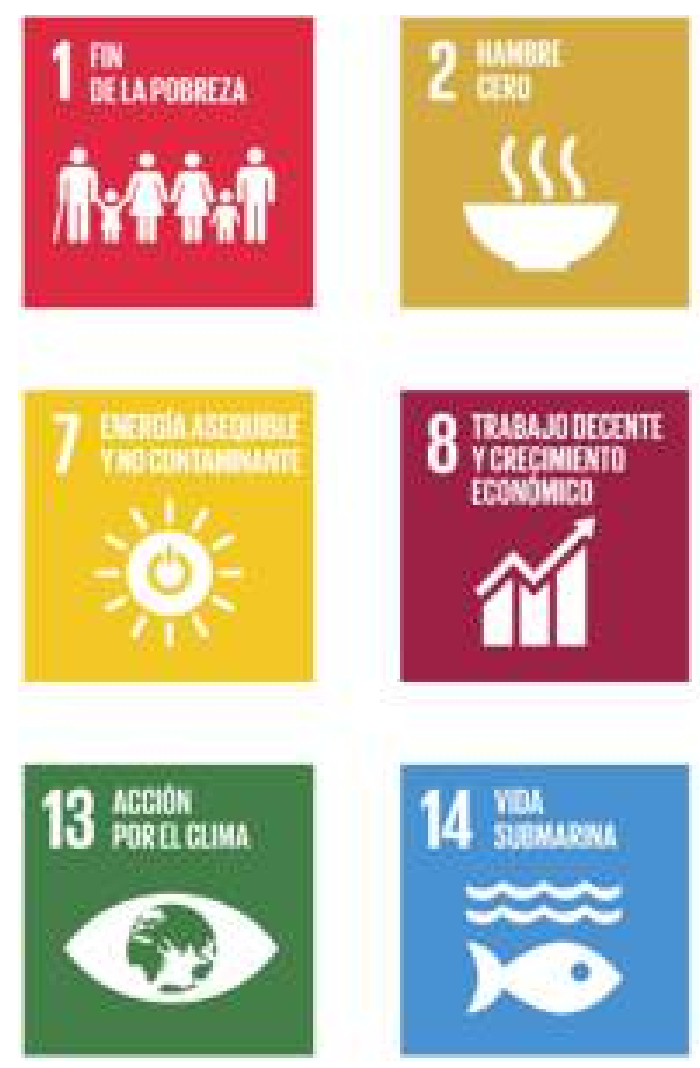

Figura 1. Objetivos de desarrollo sostenible ${ }^{1}$ Fuente: Naciones Unidas, s. f. y las necesidades de las personas que en él habitan ya no pueden esperar. Una de cada nueve personas en el mundo está subalimentada y el total en esta condición está alrededor de los 795 millones; cada año mueren más de seis millones de niños antes de haber cumplido los cinco años y una de cada cinco personas no tiene acceso a la electricidad (Pacto Mundial Red España, 2017).

\begin{abstract}
Sabemos que las empresas con visión de futuro están tomando la iniciativa transformando sus modelos comerciales para el desarrollo sostenible, y que solo hemos abordado de manera superficial las posibilidades de inversiones del sector privado basadas en la ética. Con los incentivos, las políticas, los reglamentos y la supervisión adecuados, podrían surgir grandes oportunidades. (Naciones Unidas, 2014, p. 9)
\end{abstract}

En ese sentido, los ODS representan para las empresas una oportunidad única al proporcionar lo siguiente:
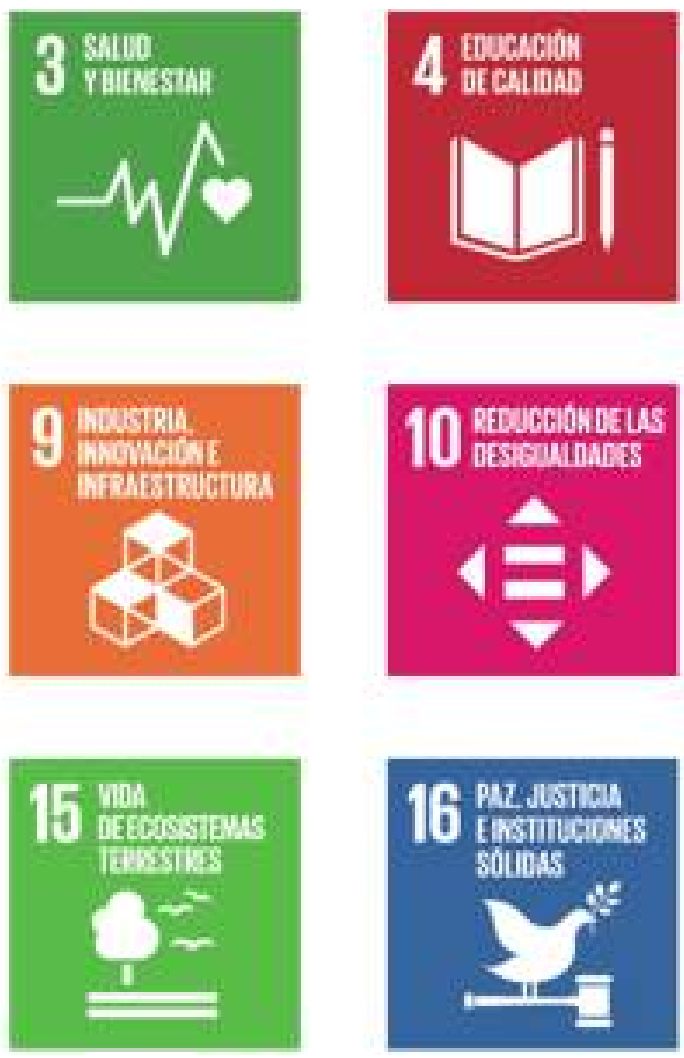

- Un marco global, con un conjunto de resultados visibles, respaldado por el gobierno, la sociedad y las empresas.

- Un horizonte a largo plazo que ofrece una dirección para la política, la inversión y la innovación, y que apunta a mercados nuevos, sin explotar en su mayoría.

- Un lenguaje común sobre cuestiones sociales, ambientales $y$ económicas, que puede ayudar a mejorar la comunicación, la coordinación y la colaboración.

Las empresas podrán descubrir nuevas oportunidades de crecimiento y reducir sus perfiles de riesgo mediante el desarrollo y la entrega de soluciones para el logro de los ODS. Las empresas pueden utilizar los ODS como un marco general para dar forma, dirigir, comunicar y reportar acerca de sus estrategias, metas y actividades, lo que les permitirá capitalizar una serie de beneficios. Estos incluyen, según refiere SDG Compass (2015), los puntos siguientes.
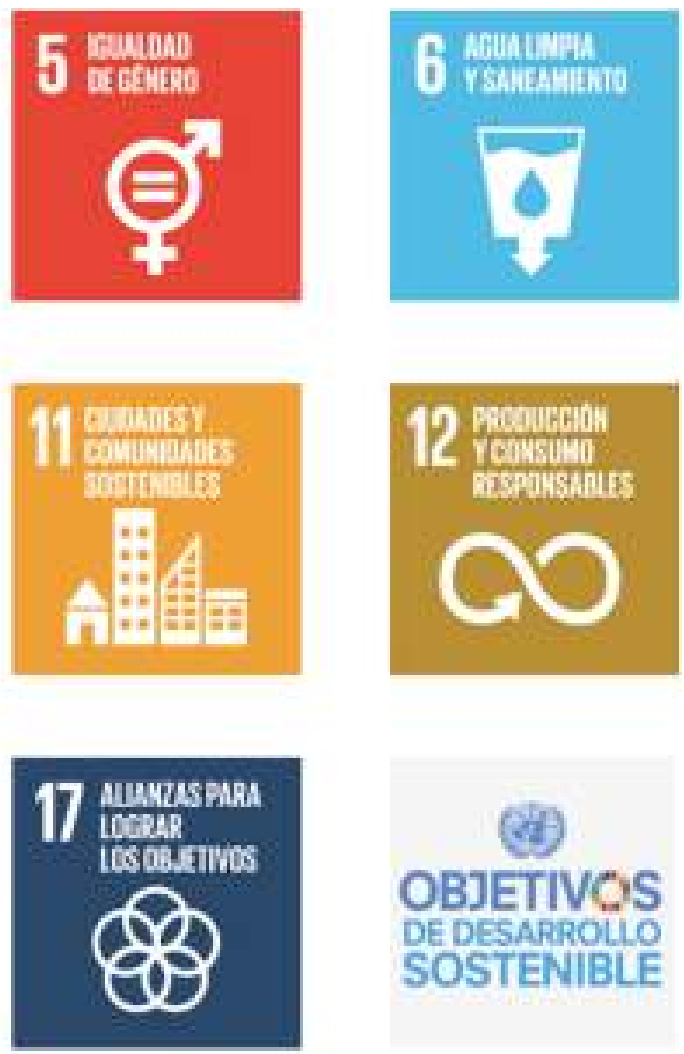

${ }^{1}$ El 25 de septiembre del 2015, los líderes mundiales agrupados en las Naciones Unidas adoptaron un conjunto de objetivos globales para erradicar la pobreza, proteger el planeta y asegurar la prosperidad para todos, como parte de una nueva agenda de desarrollo sostenible. Cada objetivo tiene metas específicas que deben alcanzarse en los próximos quince años. 
1. Identificar futuras oportunidades de negocio. Los retos mundiales de desarrollo sostenible ya representan oportunidades de mercado para aquellas empresas capaces de desarrollar y entregar soluciones distintas y efectivas; como estas:

- Las tecnologías innovadoras para aumentar la eficiencia energética, las energías renovables, el almacenamiento de energía, los edificios verdes y el transporte sostenible.

- Satisfacer las necesidades de los grandes mercados inexplotados a través de productos y servicios que pueden mejorar la vida de los 4000 millones de personas que actualmente viven en pobreza (incluidas las asistencias en salud, educación, energía, finanzas y las TIC).

\section{Mejorar el valor de la sostenibilidad} corporativa. Al integrar consideraciones de sostenibilidad a través de la cadena de valor, las empresas pueden proteger y crear valor para ellas mismas; por ejemplo, a través del incremento en ventas, el desarrollo de nuevos segmentos del mercado,

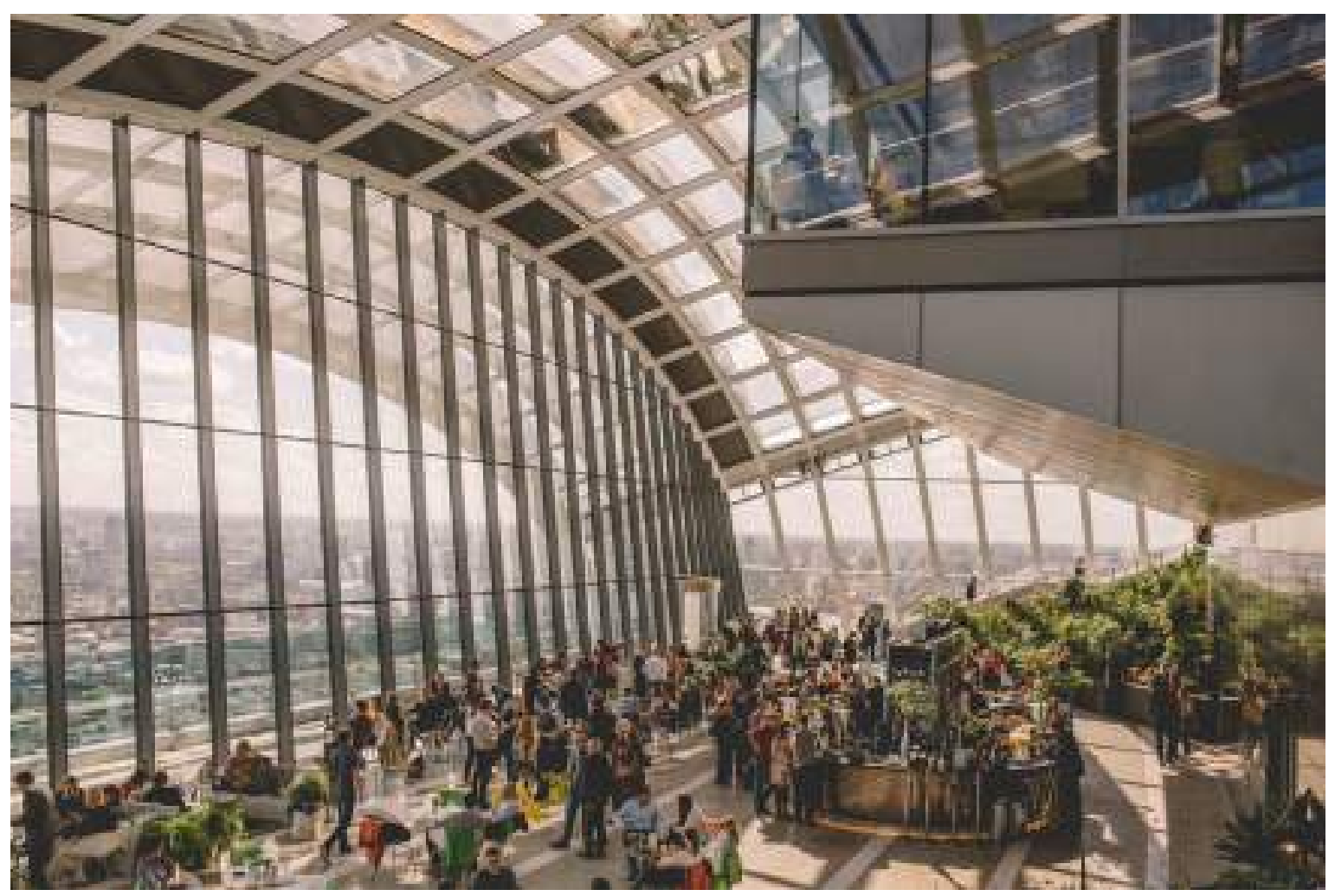

Figura 2. Equilibrio ecológico

Fuente: Negative Space, s. f. el fortalecimiento de la marca, la mejora de la eficiencia operativa, los estímulos para la innovación de productos y la reducción de la rotación de personal.

\section{Fortalecer las relaciones con las} partes interesadas y mantenerse al día con el desarrollo de políticas. Los ODS reflejan las expectativas de las partes interesadas, así como la futura dirección de la política pública en los ámbitos internacional, nacional y regional. Las empresas que alinean sus prioridades con los ODS pueden mejorar su relación con sus clientes, empleados y otras partes interesadas, mientras que aquellos que no lo hacen están expuestos a crecientes riesgos legales y de reputación.

4. Estabilizar las sociedades y los mercados. No pueden existir empresas exitosas en sociedades fracasadas. Invertir en el logro de los ODS apoya los pilares del éxito empresarial; su implementación permitirá, entre otras cosas, sacar a miles de millones de personas de la pobreza y, de esta manera, ayudar a crecer los mercados de consumo en todo el mundo.
5. Usar un lenguaje común y tener un propósito compartido. Los ODS definen un marco de acción común y un lenguaje que ayudará a las empresas a comunicar de manera más coherente y eficiente su impacto y desempeño a las partes interesadas. Pueden facilitar también la creación de alianzas más eficaces con los gobiernos, las organizaciones de la sociedad civil y con otras empresas, debido a que proporcionan un sentido unificado de las prioridades y los propósitos en todas las dimensiones del desarrollo sostenible.

\section{Los ODS y las empresas, en cifras}

Según un estudio de la Red Española del Pacto Mundial, basado en el análisis de las memorias de sostenibilidad del 2016, el $97 \%$ de las empresas del IBEX $35^{2}$ afirman ya tener presentes los ODS en su estrategia de negocio; es decir, que ya han trabajado la integración de los ODS en su empresa o planean trabajarla a corto plazo (Pacto Mundial Red España, 2017).

En la figura 4, se muestra cuáles son los ODS que tienen prioridad en la estrategia de las empresas españolas (IBEX 35). En los primeros lugares figuran aquellos con temáticas en las que el sector privado tiene algún tipo de experiencia previa. Por ejemplo, en cuestiones de igualdad de género (ODS 5) o en relación con la salud (ODS 3), las empresas llevan a cabo medidas en materia de seguridad y salud laboral. En relación con el cambio climático (ODS 13), muchas empresas cuentan con acciones encaminadas a reducir la huella de carbono.

Sin embargo, se ve que los objetivos menos trabajados son aquellos más enfocados a la acción social

\footnotetext{
${ }^{2}$ EI IBEX 35 es el principal índice bursátil de referencia de la bolsa española. Está elaborado por las bolsas y los mercados españoles (BME), y formado por las 35 empresas con mayor liquidez que cotizan en el Sistema de Interconexión Bursátil Electrónico (SIBE) en las cuatro bolsas españolas (Madrid, Barcelona, Bilbao y Valencia).
} 
(ODS 1 y ODS 2) o a temáticas menos vinculadas a los negocios (ODS 14, "Vida submarina", y ODS 16 "Paz, justicia e instituciones sólidas"). El reto es encontrar formas en las que el sector privado pueda contribuir a estos objetivos tradicionalmente más enfocados a la sociedad civil o a las instituciones públicas.

A nivel internacional, se muestra un ligero cambio en relación con las prioridades de las empresas españolas. En los primeros lugares de prioridad, está el objetivo 8, “Trabajo decente y crecimiento económico", y el objetivo 17, "Alianzas para lograr los objetivos", mientras que, según la misma estadística, los ODS 2, "Hambre cero", y 14, "Vida submarina", son los que menos prioridad tienen a nivel internacional (Pacto Mundial Red España, 2017).

\section{Los riesgos de la inacción ante los} objetivos de desarrollo sostenible

Según refiere la guía de los CEO sobre los ODS, es importante que las empresas sean conscientes de sus obligaciones fundamentales.

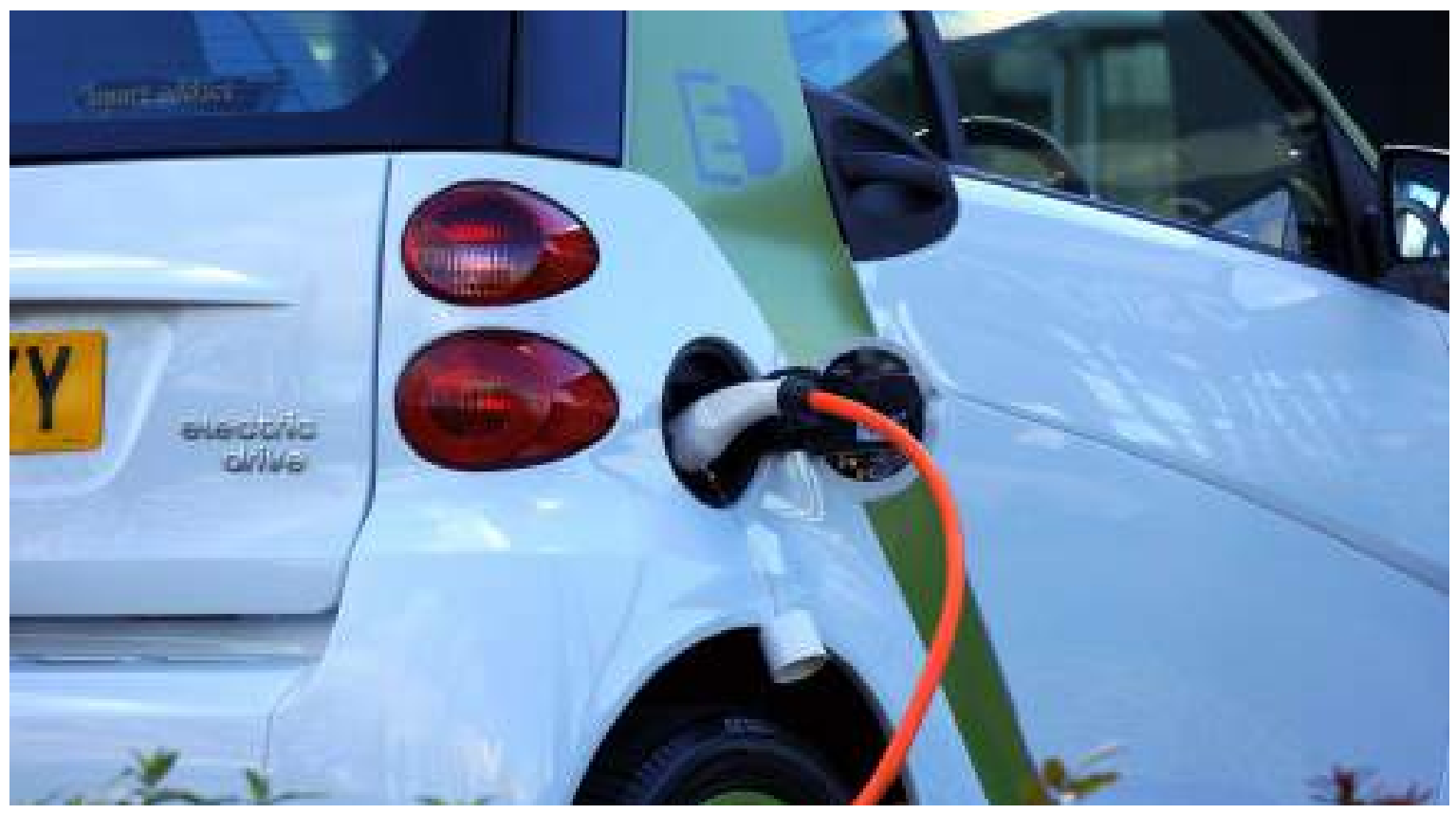

Figura 3. Energía limpia

Fuente: Mikes Photos, s. f.

El punto de partida para todas las empresas debería ser garantizar que sus actividades no obstaculicen el cumplimiento de esta agenda; más bien, su prioridad debería ser abordar los impactos sociales negativos, especialmente las empresas relacionadas con los derechos humanos, como indica el World Business Council for Sustainable Development (2017). No asumir este reto no significa que las empresas desaparezcan, pero surgen aspectos a considerar, como los siguientes:
1. Riesgo regulatorio. Los ODS reflejan la dirección que tomarán las políticas en el futuro a nivel internacional, nacional y regional. Si no se integran de forma estratégica, esto podría conllevar un riesgo regulatorio a largo plazo.

2. Nuevas reglas en los mercados. Las empresas con visión de futuro están llevando adelante nuevos modelos de negocio disruptivos que amenazan con cambiar radicalmente los mercados.

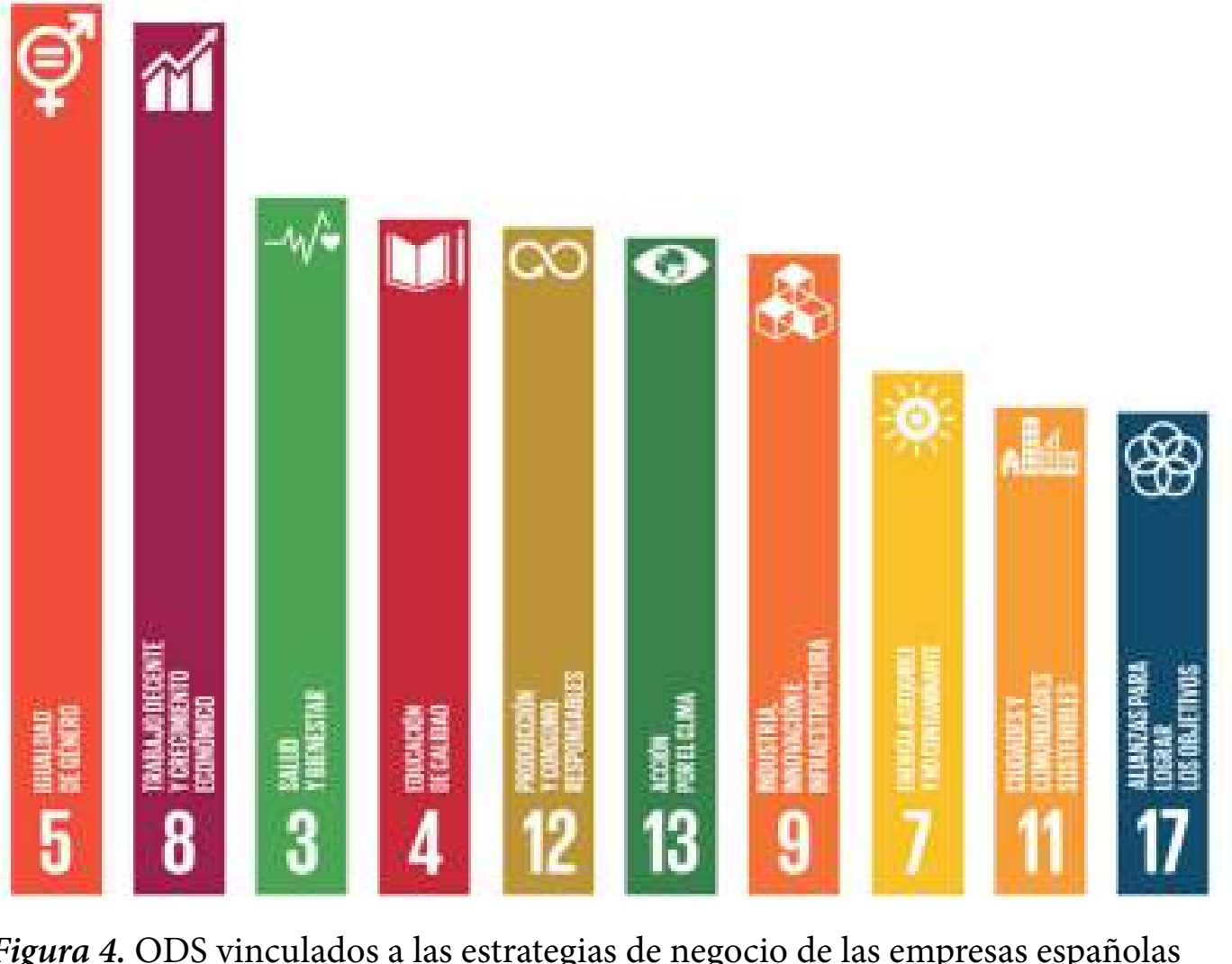

Figura 4. ODS vinculados a las estrategias de negocio de las empresas españolas Fuente: Pacto Mundial Red España, 2017 


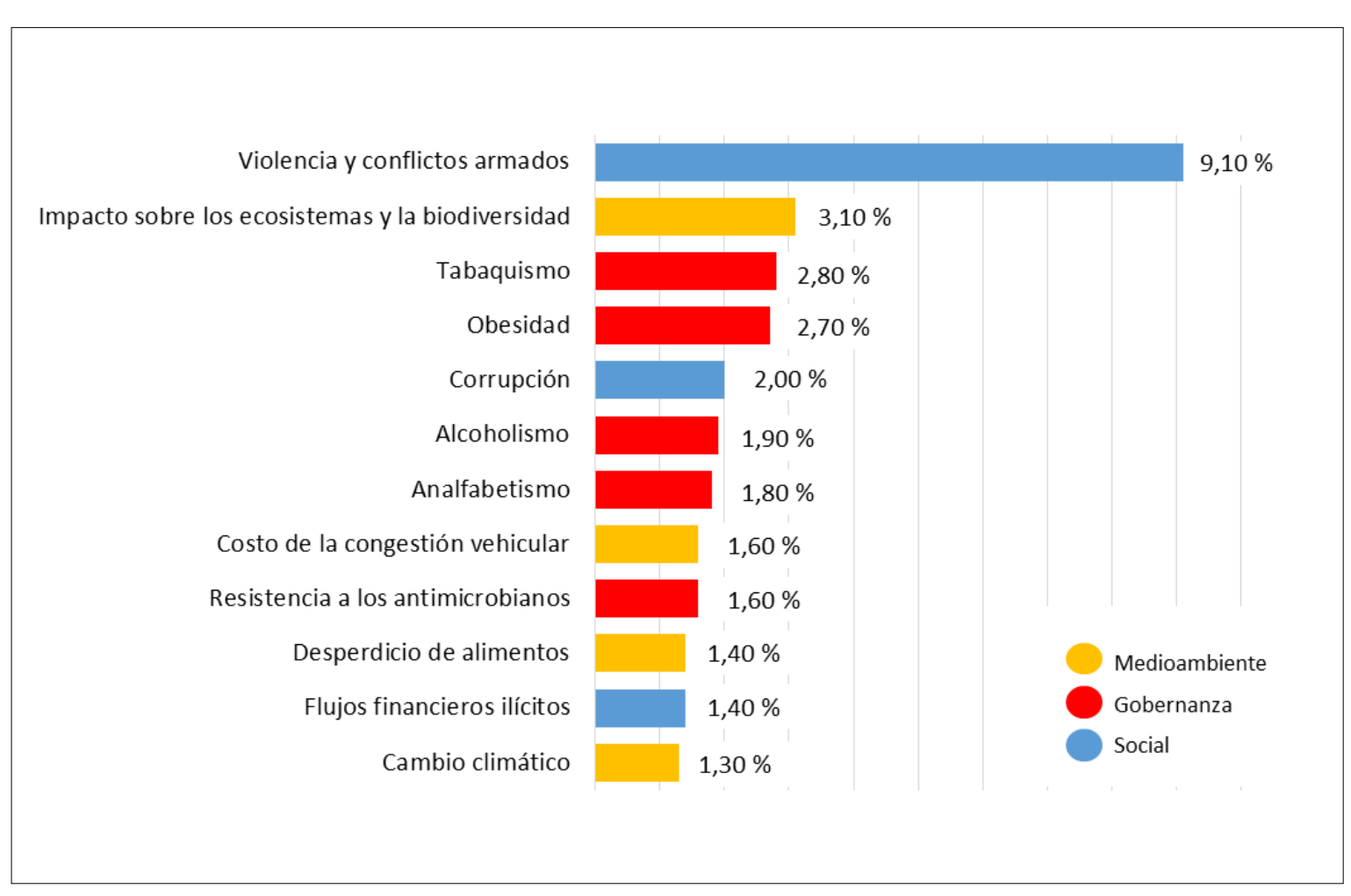

Figura 5. Estimado anual y global de los impactos económicos directos asociados con problemas globales seleccionados, con porcentaje del PBI mundial del 2014.

Fuente: Business and Sustainable Development Commission, 2017

\section{Reducción de la licencia social para} operar. El último informe global sobre confianza en las empresas de Edelman muestra un descenso de dos dígitos en la credibilidad de los CEO en un $80 \%$ de los países a lo largo del último año.

\section{Costos de la inacción}

Las deficiencias del modelo económico actual se unen a la larga lista de problemáticas sociales y ambientales que frenan nuestras perspectivas de crecimiento para el futuro; estas problemáticas suponen un aumento de los costos empresariales y, a largo plazo, hacen del mundo un lugar menos viable para hacer negocios.

En la figura 5 se muestra un estimado de los impactos económicos directos asociados con problemas globales, sobre la base del porcentaje del PBI mundial del 2014.

\section{Es hora de aprovechar las oportunidades}

Con el desarrollo sostenible se generan nuevas oportunidades y aumento de eficiencia en las empresas, se promueve la innovación y se mejora la reputación. Con el prestigio de la sostenibilidad, se atrae y retiene a los empleados, a los consumidores y a los clientes e inversores, y se garantiza su aptitud para operar.

Según refiere la Business and Sustainable Development Commission (2017), el alcanzar los objetivos de desarrollo sostenible permitirá por lo menos la creación de 380 millones de nuevos empleos para el 2030, casi el $90 \%$ de ellos en los países en desarrollo.

¿Cuáles son las oportunidades de negocio que tendrían las empresas? La tabla 1 muestra las sesenta mayores oportunidades de mercado relacionadas con el logro de los objetivos de desarrollo sostenible.

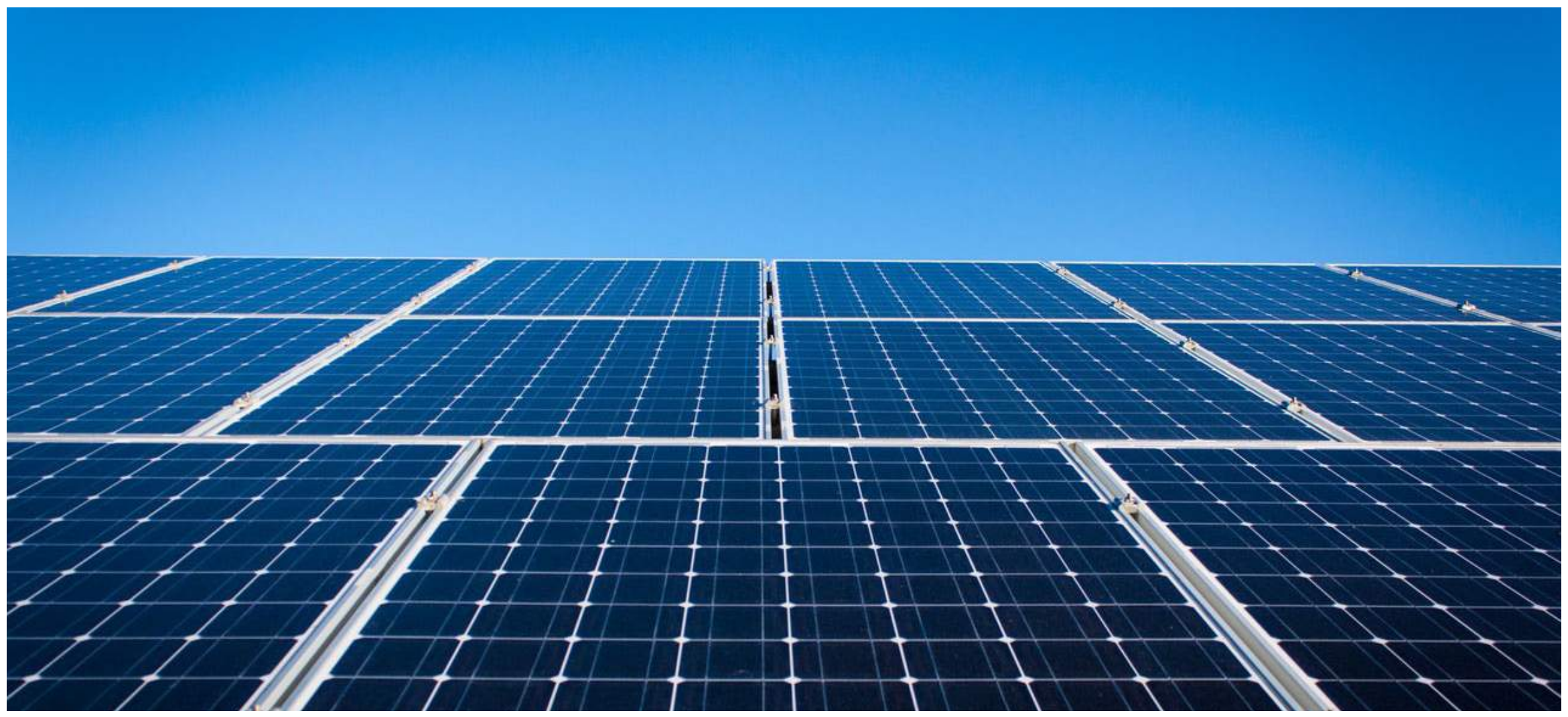

Figura 6. Energías renovables

Fuente: Carl Attard, s. f. 


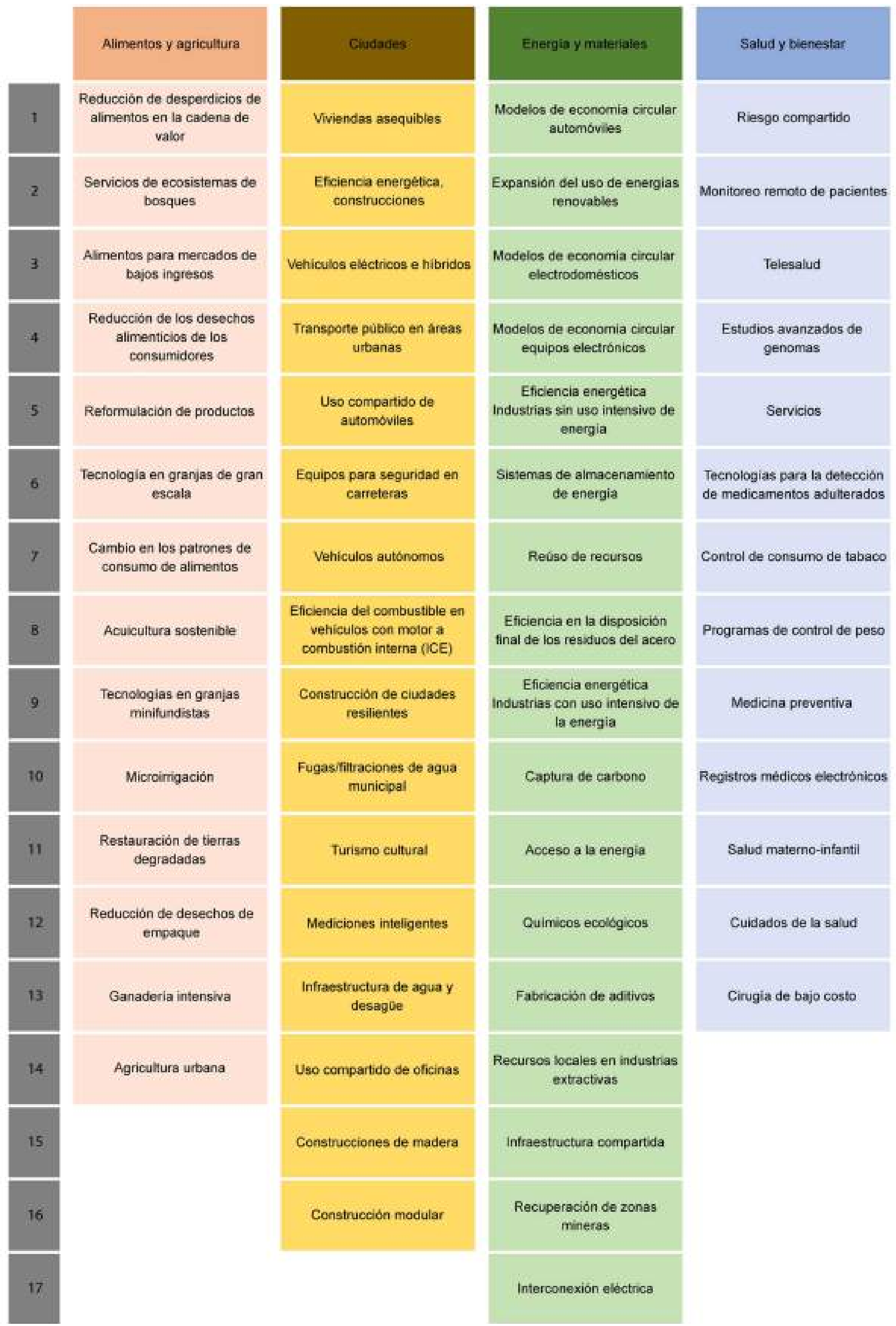

Tabla 1. Las sesenta mayores oportunidades de mercado relacionadas con el logro de los objetivos de desarrollo sostenible Fuente: Business and Sustainable Development Commission, 2017 


\section{A manera de conclusión}

Dado que los ODS conforman la agenda global para el desarrollo de las sociedades, estos permitirán que las empresas líderes demuestren de qué manera sus negocios contribuyen a avanzar en el desarrollo sostenible, tanto ayudando a minimizar los impactos negativos como maximizando los impactos positivos sobre las personas y el planeta.

Los objetivos de desarrollo sostenible aceptan y reconocen el papel del sector privado, sin discriminar el tamaño de la organización o su giro de actividad, dándole el rol de motor de la productividad, el crecimiento económico inclusivo y la creación de empleo.
Como se ha podido ver en este breve artículo, las empresas deben echar mano de la creatividad y la innovación para resolver los problemas relacionados con el desarrollo sostenible. Además, deberían adoptar prácticas propias de la responsabilidad social, relacionadas con el empleo digno, el empoderamiento de la mujer, la inversión en investigación y desarrollo, la protección del medioambiente, la transparencia y la rendición de cuentas, que van más allá del ámbito puramente económico. Un mensaje final para los directores de empresas: no es el momento de ser conservadores, sino de adoptar desafíos.

A mediados del siglo $\mathrm{xx}$, vimos nuestro planeta desde el espacio por primera vez. Los historiadores pueden eventualmente encontrar que esta imagen tuvo un mayor impacto en el pensamiento que la revolución copernicana del siglo XvI, que trastornó la autoimagen humana al revelar que la Tierra no es el centro del universo. Desde el espacio, vemos una bola pequeña y frágil dominada no por la actividad humana y la infraestructura, sino por un patrón de nubes, océanos, bosques y suelos. La incapacidad de la humanidad de encajar sus actividades en ese patrón está cambiando ese sistema planetario. Muchos de esos cambios van acompañados de peligros que amenazan la vida. Esta nueva realidad, de la que no hay escapatoria, debe ser reconocida y gestionada. (World Commission on Environment and Development, 1987)

\section{Referencias}

Business and Sustainable Development Commission. (2017). Better business. Better world. Recuperado de http://report.businesscommission.org/uploads/BetterBiz-BetterWorld_170215_012417.pdf

Carl Attard. (s. f.). [Fotografía]. Recuperado de https:// www.pexels.com/photo/alternative-alternative-energy-blue-eco-411592/

Kaboompics.com. (s. f.). Young grain. Recuperado de https://www.pexels.com/photo/young-grain-5865/

Mikes Photos. (s. f.). [Fotografía]. Recuperado de https:// www.pexels.com/photo/alternative-auto-automobile-battery-110844/

Naciones Unidas. (s. f.). Objetivos de desarrollo sostenible. Recuperado de http://www.un.org/sustainabledevelopment/es/objetivos-de-desarrollo-sostenible/

Negative Space. (s. f.). [Fotografía]. Recuperado de https://www.pexels.com/photo/party-glass-architecture-windows-34173/

Organización de las Naciones Unidas. (s. f.). Objetivos de desarrollo sostenible. Recuperado de http://www.un.org/sustainabledevelopment/es/objetivos-de-desarrollo-sostenible/

Organización de las Naciones Unidas. (1987). Informe de la Comisión Mundial sobre el Medio Ambiente y el Desarrollo. Recuperado de https://undocs.org/es/A/42/427
Organización de las Naciones Unidas. (2014). El camino hacia la dignidad para 2030: acabar con la pobreza y transformar vidas protegiendo el planeta. Informe de síntesis del Secretario General sobre la agenda de desarrollo sostenible después de 2015. Recuperado de http://www.un.org/en/ga/search/view_ doc.asp?symbol=A/69/700\&referer=http://www.un.org/en/ documents/\&Lang=S

Red Española del Pacto Mundial. (2017). Empresas españolas y ODS: Oportunidades y desafíos. Recuperado de http:// www.pactomundial.org/wp-content/uploads/2017/04/Empresas_y_ODS_PM_20170405.pdf

Scott Webb. (s. f.). White and blue building during daytime. Recuperado de https://www.pexels.com/photo/building-modern-glass-tall-27406/

SDG Compass. (s. f.). La guía para la acción empresarial en los ODS. Recuperado de https://sdgcompass.org/wp-content/ uploads/2016/06/SDG_Compass_Spanish-one-pager-view.pdf

World Business Council for Sustainable Development. (2017). Guía para CEOs sobre los objetivos de desarrollo sostenible. Recuperado de http://peru2021.org/wp-content/ uploads/2017/10/Gui\%CC\%81a-CEO-sobre-ODS.pdf 